\title{
THE GENERALIZED HARMONIC SERIES AS A FUNDA- MENTAL PRINCIPLE OF SOCIAL ORGANIZATION
}

\author{
By George KingsLey ZipF \\ Harvard University
}

The present paper is a preliminary report of observations.

Let us call the following equation the equation of the generalized harmonic series: $A S n=\frac{A}{1}+\frac{A}{2^{p}}+\frac{A}{3^{p}}+\cdots \cdots \cdot \ldots+\frac{A}{n^{p}}$

The writer has observed that this equation, with the exponent $\mathrm{p}=1$, describes the distribution of communities larger than 2,500 inhabitants in the United States for 1930, when communities are arranged in the order of decreasing size (in which $A$ equals the size of the largest community). Furthermore he has observed that the distributions of communities in India, Germany, and other countries, approximate the equation of the generalized harmonic series, with values of $p$ lying between 0 and 1 . In view of these observations, the author concludes that what we call a "nation" is in fact a bio-social entity, analogous to a colony of ants, or of bees, or of termites.

Moreover the writer has observed that the national distribution of monetary incomes in the United States and other countries tends to approximate the equation of the generalized harmonic series, and similarly the distribution of corporation assets (as well as other distributions). The author has also noted a connection between these formulae on the one hand, and what we might call the psychology of culture and of economics on the other.

The author reserves these problems and this manner of analysis of this type of data for his own more comprehensive treatment in an extensive study to be published in the immediate future under the title of THE LAWS OF NATIONAL FORCES: MAN'S PLACE IN NATURE'S BALANCE. And in this more comprehensive treatment will be presented a qualitative discussion of checks and balances which seem to bring about and retain these distributions.

Copyright 1940

All rights reserved to the author. 